\title{
AN ABSTRACT INVERSE PROBLEM ${ }^{1}$
}

\author{
M. CHOULLI \\ Université de France-Comté \\ Laboratory de Mathématiques \\ 25030, route de Gray, Besançon cédex \\ FRANCE
}

\begin{abstract}
In this paper we consider an inverse problem that corresponds to an abstract integrodifferential equation. First, we prove a local existence and uniqueness theorem. We also show that every continuous solution can be locally extended in a unique way. Finally, we give sufficient conditions for the existence and a stability of the global solution.
\end{abstract}

Key words: inverse problem, abstract integrodifferential equation, existence, uniqueness, stability.

AMS (MOS) subject classification: $\quad 35 R$.

\section{INTRODUCTION}

Let $X, Y$ be two Banach spaces, and let $A: D(A) \subset X \rightarrow X$ be a linear operator. Let $T>0, F_{1}, F_{2}:[0, T] \times X \times Y \rightarrow X, L: X \rightarrow Y, v:[0, T] \rightarrow Y$, and $x \in X$ be given data.

We consider the following problem: find $(u, p):[0, T] \rightarrow X \times Y$ such that

$$
\begin{aligned}
& u^{\prime}(t)=A u(t)+F_{1}(t, u(t), p(t))+\int_{0}^{t} F_{2}(s, u(s), p(t-s)) d s, 0 \leq t \leq T, \\
& u(0)=x, \\
& L u(t)=v(t), 0 \leq t \leq T .
\end{aligned}
$$

Such a problem has been considered previously by Prilepko, Orlovskii in [6,7], Lorenzi, Sinestrari in [4], and the author in [1].

The local existence and uniqueness result is obtained by Prilepko, Orlovskii for the case $F_{2}=0$, and by Lorenzi, Sinestrari for the case $Y$ is a subspace of $L(X), F_{1}(t, u, p)=p B x$, and $F_{2}(t, u, p)=p B u$, where $B$ is some given linear operator in $X$. The stability problem has been studied by Lorenzi and Sinestrari in [5].

In [1] the author treats the case of $Y=C[0, T]^{n}(n \geq 1), F_{1}\left(t, u,\left(p_{1}, \ldots, p_{n}\right)\right)=\sum_{1}^{n} p_{i} y_{i}, y_{i}$ in $X(1 \leq i \leq n)$ and $F_{2}=0$. Then a global existence and uniqueness theorem is obtained.

\footnotetext{
${ }^{1}$ Received: January 1990; Revised: October 1990.
} 
The present work is concerned with a generalization of those results.

Throughout this paper we assume:

(H1) $A$ is a closed linear operator with a dense domain generating a strongly continuous semigroup $e^{A t}$. Without loss of generality, we suppose that $e^{A t}$ is equibounded:

$$
\left\|e^{A t}\right\| \leq M, t \geq 0 \text { for some } M \geq 1
$$

(H2) $x \in D(A)$,

(H3) $L \in L(X, Y)$,

(H4) $\quad v \in C^{1}([0, T]: Y)$, and $v(0)=L x$.

$(H 5,1) \quad F_{1}$ and $A F_{1}$ are continuous in $[0, T] \times D(A) \times Y$.

For each $r>0$, there exist positive continuous real valued functions $g_{1, i}(r, \cdot), i=0,1$ such that

$(H 5,2) \quad\left\|F_{1}\left(t, u_{1}, p_{1}\right)\right\|_{D(A)} \leq g_{1,0}(r, t)$,

$(H 5,3) \quad\left\|F_{1}\left(t, u_{1}, p_{1}\right)-F_{1}\left(t, u_{2}, p_{2}\right)\right\|_{D(A)} \leq g_{1,1}(r, t)\left(\left\|u_{1}-u_{2}\right\|_{D(A)}+\left\|p_{1}-p_{2}\right\|_{Y}\right)$,

for each $\left(u_{i}, p_{i}\right) \in\left\{(u, p) \in D(A) \times Y,\|u\|_{D(A)}+\|p\|_{Y} \leq r\right\}, i=1,2$, and $t \in[0, T]$.

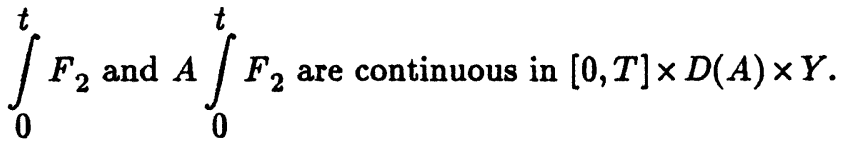

For each $r>0$, there exist positive continuous real valued functions $g_{2, i}(r, \cdot), i=0,1$, such that

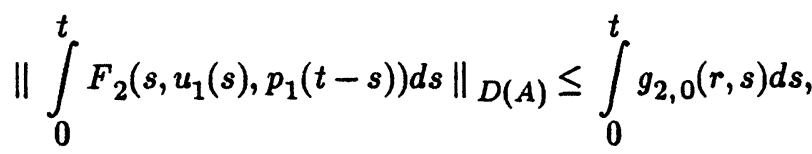

$$
\begin{aligned}
& \left\|\int_{0}^{t}\left(F_{2}\left(s, u_{1}(s), p_{1}(t-s)\right)-F_{2}\left(s, u_{2}(s), p_{2}(t-s)\right)\right) d s\right\|_{D(A)} \\
& \quad \leq \int_{0}^{t} g_{2,1}(r, s)\left(\left\|u_{1}(s)-u_{2}(s)\right\|_{D(A)}+\left\|p_{1}(s)-p_{2}(s)\right\|_{Y}\right) d s
\end{aligned}
$$

for each $\left(u_{i}, p_{i}\right) \in\left\{(u, p) \in C([0, T]: D(A) \times Y) \sup _{0 \leq s \leq t}\left(\|u(s)\|_{D(A)}+\|p(s)\|_{Y}\right) \leq r\right\}, i=1,2$, and $t \in[0, T]$.

There exist continuous function $H_{1}:[0, T] \times Y \times Y \rightarrow Y$ with the following properties. For each $r>0$ there exist positive continuous real valued functions $C(r, \cdot)$ such that

$(H 7,1) \quad\left\|H_{1}\left(t, u_{1}, p_{1}\right)-H_{1}\left(t, u_{2}, p_{2}\right)\right\|_{Y} \leq C(r, t)\left(\left\|u_{1}-u_{2}\right\|_{D(A)}+\left\|p_{1}-p_{2}\right\|_{Y}\right)$, for each $\left(u_{i}, p_{i}\right) \in\left\{(u, p) \in Y \times Y,\|u\|_{Y}+\|p\|_{Y} \leq r\right\}, i=1,2$, and $t \in[0, T]$.

$K: p \rightarrow H_{1}(t, v(t), p)$ has an inverse $\Phi(t, \cdot)$ continuous map $t \rightarrow \Phi(t, w)$, and there exist positive continuous real valued function $k$, such that 
$(H 7,2) \quad\left\|\Phi\left(t, w_{1}\right)-\Phi\left(t, w_{2}\right)\right\|_{Y}, t \in[0, T], w_{i} \in Y, i=1,2$.

$(H 7,3) \quad L F_{1}(t, u, p)=H_{1}(t, L u, p),(u, p) \in D(A) \times Y$, and $t \in[0, T]$.

\section{EXISTENCE OF THE LOCAL SOLUTION}

In this section we prove that the local solution of our inverse problem is obtained by a fixed point theorem. Let

$$
\begin{gathered}
a(t)=M\|x\|_{D(A)}+\|\Phi(t, 0)\|_{Y}+k(t)\left\|v^{\prime}(t)-L e^{A t} A x\right\|_{Y}, t \in[0, T], r_{0}=2 \underset{\substack{\text { sup } \\
0 \leq t \leq T}}{a(t),} \\
g_{i}\left(r_{0}, t, s\right)=M(1+k(t)\|L\|)\left(g_{1, i}\left(r_{0}, s\right)+(t-s) g_{2, i}\left(r_{0}, s\right)\right)+k(t)\|L\| g_{2, i}\left(r_{0}, s\right), \quad 0 \leq s \leq t \leq T,
\end{gathered}
$$
$i=0,1$, and let $T_{0} \in[0, T]$ be such that

$$
T_{0} \underset{0 \leq s \leq t \leq T}{\sup } g_{0}\left(r_{0}, t, s\right) \leq \frac{r_{0}}{2}, \text { and } T_{0} \underset{0 \leq s \leq t \leq T}{\sup } g_{1}\left(r_{0}, t, s\right)=\gamma<1 .
$$

Let $Z\left(T_{0}\right)=C\left(\left[0, T_{0}\right]: D(A) \times Y\right)$ equipped with the norm

$$
\|(u, p)\|_{Z\left(T_{0}\right)}=\sup _{0 \leq t \leq T_{0}}\left(\|u(t)\|_{D(A)}+\|p(t)\|_{Y}\right) .
$$

Then, we define the mapping

$$
\Psi: Z\left(T_{0}\right) \rightarrow Z\left(T_{0}\right):(u, p) \rightarrow(U, P)
$$

where

$$
\begin{gathered}
U(t)=e^{A t} x+\int_{0}^{t} e^{A(t-s)} F_{1}(s, u(s), p(s)) d s \\
+\int_{0}^{t} e^{A(t-s)} \int_{0}^{s} F_{2}(\sigma, u(\sigma), p(s-\sigma)) d \sigma d s \\
P(t)=\Psi\left(t, v^{\prime}(t)-L e^{A t} A x-\int_{0}^{t} L F_{2}(s, u(s), p(t-s)) d s\right. \\
-\int_{0}^{t} L e^{A(t-s)} A F_{1}(s, u(s), p(t-s)) d s \\
\left.\quad s e^{A(t-s)} A \int_{0}^{s} F_{2}(\sigma, u(\sigma), p(s-\sigma)) d \sigma d s\right), 0 \leq t \leq T_{0} .
\end{gathered}
$$

Proposition 1. There exists a unique $\left(u_{0}, p_{0}\right)$ in $B\left(r_{0}, T_{0}\right)$ satisfying $\left(u_{0}, p_{0}\right)=\Psi\left(u_{0}, p_{0}\right)$, where $B\left(r_{0}, T_{0}\right)$ denotes the closed ball of $Z\left(T_{0}\right)$ with the center 0 and radius $r_{0}$. 
Proof. We claim that $\Psi$ is a strict contraction from $B\left(r_{0}, T_{0}\right)$ into itself. Hence, according to the fixed point theorem, there is a unique $\left(u_{0}, p_{0}\right)$ in $B\left(r_{0}, T_{0}\right)$ such that $\left(u_{0}, p_{0}\right)=\Psi\left(u_{0}, p_{0}\right)$. Let $\left(u_{i}, p_{i}\right)$ in $B\left(r_{0}, T_{0}\right),\left(U_{i}, P_{i}\right)=\Psi\left(u_{i}, p_{i}\right), i=1,2$, and $t$ in $\left[0, T_{0}\right]$.

We have then

Using (H5) and (H6) we obtain

$$
\begin{gathered}
\left\|U_{1}(t)\right\|_{D(A)} \leq M\|x\|_{D(A)}+M \int_{0}^{t}\left\|F_{1}(s, u(s), p(s))\right\|_{D(A)} d s \\
+M \int_{0}^{t}\left\|\int_{0}^{s} F_{2}(\sigma, u(\sigma), p(s-\sigma)) d \sigma\right\|_{D(A)} d s .
\end{gathered}
$$

$$
\begin{aligned}
& \left\|U_{1}(t)\right\|_{D(A)} \leq M\|x\|_{D(A)}+M \int_{0}^{t} g_{1,0}\left(r_{0}, s\right) d s+M \int_{0}^{t} \int_{0}^{s} g_{2,0}\left(r_{0}, \sigma\right) d \sigma d s \\
& \leq M\|x\|_{D(A)}+M \int_{0}^{t}\left(g_{1,0}\left(r_{0}, s\right)+(t-s) g_{2,0}\left(r_{0}, s\right)\right) d s .
\end{aligned}
$$

From $(H 7,2)$ we deduce

$$
\begin{gathered}
\left\|P_{1}(t)\right\|_{Y} \leq\|\Phi(t, 0)\|_{Y}+k(t) \| v^{\prime}(t)-L e^{A t} A x \\
-\int_{0}^{t} L F_{2}(s, u(s), p(t-s)) d s-\int_{0}^{t} L e^{A(t-s)} A F_{1}(s, u(s), p(t-s)) d s \\
\left.-\int_{0}^{t} L e^{A(t-s)} A \int_{0}^{s} F_{2}(\sigma, u(\sigma), p(s-\sigma)) d \sigma d s\right) \|_{Y} .
\end{gathered}
$$

Hence

$$
\begin{aligned}
\left\|P_{1}(t)\right\|_{Y} \leq & \|\Phi(t, 0)\|_{Y}+k(t)\left\|v^{\prime}(t)-L e^{A t} A x\right\|+\|L\| k(t) \int_{0}^{t} g_{2,0}\left(r_{0}, s\right) d s \\
& +M\|L\| k(t) \int_{0}^{t}\left(g_{1,0}\left(r_{0}, s\right)+(t-s) g_{2,0}\left(r_{0}, s\right)\right) d s .
\end{aligned}
$$

Thus

$$
\begin{aligned}
\left\|U_{1}(t)\right\|_{D(A)}+\left\|P_{1}(t)\right\|_{Y} & \leq\|x\|_{D(A)}+\|\Phi(t, 0)\|_{Y}+k(t)\left\|v^{\prime}(t)-L e^{A t} A x\right\| \\
& +\|L\| k(t) \int_{0}^{t} g_{2,0}\left(r_{0}, s\right) d s \\
+M(1+\|L\| k(t)) \int_{0}^{t}\left(g_{1,0}\left(r_{0}, s\right)+(t-s) g_{2,0}\left(r_{0}, s\right)\right) d s & \\
& \leq a(t)+\int_{0}^{t} g_{1}\left(r_{0}, t, s\right) d s .
\end{aligned}
$$


This implies that

$$
\left\|\left(U_{1}, P_{1}\right)\right\|_{Z\left(T_{0}\right)} \leq r_{0}
$$

On the other hand, in the same way as above, it is easily seen that

$$
\begin{gathered}
\left\|U_{1}(t)-U_{2}(t)\right\|_{D(A)}+\left\|P_{1}(t)-P_{2}(t)\right\|_{Y} \\
\leq \int_{0}^{t} g_{2}\left(r_{0}, t, s\right)\left(\left\|u_{1}(s)-u_{2}(s)\right\|_{D(A)}+\left\|p_{1}(s)-p_{2}(s)\right\|_{Y}\right) d s \\
\leq \gamma \sup _{0 \leq s \leq t}\left(\left\|u_{1}(s)-u_{2}(s)\right\|_{D(A)}+\left\|p_{1}(s)-p_{2}(s)\right\|_{Y}\right) d s .
\end{gathered}
$$

It follows that

$$
\left\|\left(U_{1}, P_{1}\right)-\left(U_{2}, P_{2}\right)\right\|_{Z\left(T_{0}\right)} \leq \gamma\left\|\left(u_{1}, p_{1}\right)-\left(u_{2}, p_{2}\right)\right\|_{Z\left(T_{0}\right)^{*}}
$$

Our claim is proven.

Proposition 2. $(u, p)$ is a solution of the inverse problem $(1)-(3)$ in $[0, T]$ iff $(u, p)=\Psi(u, p)$.

Proof. It is well known that the solution of Cauchy problem (1) and (2) is given by $u(t)=U(t)$. Therefore, it suffices to show

for each $t$ in $[0, T]$.

$$
L u(t)=v(t) \text { iff } p(t)=\Psi\left(t, v^{\prime}(t)-\int_{0}^{t} L F_{2}(s, u(s), p(t-s)) d s-L A u(t)\right)
$$

First, we differentiate $L u(t)=v(t)$ to obtain

Hence

$$
L u^{\prime}(t)=L\left\{A u(t)+F_{1}(t, u(t), p(t))+\int_{0}^{t} F_{2}(s, u(s), p(t-s)) d s\right\}=v^{\prime}(t) .
$$

Using $(H 7,2)$ we get

$$
\begin{gathered}
H_{1}(t, v(t), p(t))=L F_{1}(t, u(t), p(t)) \\
=v^{\prime}(t)-\int_{0}^{t} L F_{2}(s, u(s), p(t-s)) d s-L A u(t) .
\end{gathered}
$$

$$
p(t)=\Psi\left(t, v^{\prime}(t)-\int_{0}^{t} L F_{2}(s, u(s), p(t-s)) d s-L A u(t)\right)
$$

Conversely, this last equality implies that 


$$
\begin{aligned}
H_{1}(t, v(t), p(t)) & =v^{\prime}(t)-L\left\{\int_{0}^{t} F_{2}(s, u(s), p(t-s)) d s-A u(t)\right\} \\
& -v^{\prime}(t)-L\left\{u^{\prime}(t)-F_{1}(t, u(t), p(t))\right\} \\
& =v^{\prime}(t)-L u^{\prime}(t)+H_{1}(t, L u(t), p(t)) .
\end{aligned}
$$

Thus

$$
\frac{d}{d t}(v(t))=H_{1}(t, v(t), p(t))-H_{1}(t, L u(t), p(t)) .
$$

Integrating and using the fact that $v(0)=L u(0)=L x$, we obtain

But, $(H 7,1)$ leads to

$$
v(t)-L u(t)=\int_{0}^{t}\left(H_{1}(s, v(s), p(s))-H_{1}(s, L u(s), p(s))\right) d s .
$$

where

$$
\|v(t)-L u(t)\|_{Y}=\int_{0}^{t} C(R, s)\|v(s)-L u(s)\|_{Y} d s
$$

$$
R=\max \left(\sup _{0 \leq t<T}\left(\|L u(t)\|_{Y}+\|p(t)\|_{Y}\right), \sup _{0 \leq t \leq T}\left(\|v(t)\|_{Y}+\|p(t)\|_{Y}\right)\right) .
$$

Hence, by using Gronwall's inequality, it follows that

$$
v(t)-L u(t)=0,0 \leq t \leq T .
$$

Now, we combine propositions 1 and 2 to deduce the following local existence and uniqueness theorem for the inverse problem (1) $-(3)$.

Theorem 1. Under the assumptions $(H 1)-(H 7)$, there exist $T_{0}$ in $[0, T]$ and $\left(u_{0}, p_{0}\right)$ in $C\left(\left[0, T_{0}\right]: D(A) \times Y\right)$ which is the unique solution of the inverse problem $(1)-(3)$ in $\left[0, T_{0}\right]$.

Remark. Theorem 1 is still valued if we add to the right side of equality (1) a function $f:[0, T] \rightarrow X$ such that $f$ and $A f$ are continuous.

\section{GLOBAL SOLUTION}

We begin this section by showing that any solution $\left(u_{0}, p_{0}\right)$ in $C\left(\left[0, T_{0}\right]: D(A) \times Y\right)$ of the inverse problem $(1)-(3)$ in $\left[0, T_{0}\right]$ can be uniquely extended to a solution in $\left[0, T_{0}+T_{1}\right]$ for some $T_{1}>0$, whenever $0<T_{0}<T$.

If $\bar{T}$ is in $\left[0, \min \left(T_{0}, T-T_{0}\right)\right]$, we consider the following inverse problem: 


$$
\begin{aligned}
& u^{\prime}(t)=A u(t)+K_{1}(t, u(t), p(t))+\int_{0}^{t} K_{2}(s, u(s), p(t-s)) d s+f(t), 0 \leq t \leq \bar{T} \\
& u(0)=x_{1}=u_{0}\left(T_{0}\right) \\
& L u(t)=w(t), 0 \leq t \leq \bar{T}
\end{aligned}
$$

where

$$
\begin{gathered}
K_{1}(t, u(t), p(t))=F_{1}\left(t+T_{0}, u(t), p(t)\right), 0 \leq t \leq \bar{T}, \\
K_{2}(s, u(s), p(t-s))=F_{2}\left(s, u_{0}(s), p(t-s)\right)+F_{2}\left(s+T_{0}, u(s), p_{0}(t-s)\right), 0 \leq t \leq \bar{T}, \\
f(t)=\int_{t}^{T_{0}} F_{2}\left(s, u_{0}(s), p_{0}\left(t+T_{0}-s\right)\right) d s, 0 \leq t \leq \bar{T}, \text { and } \\
w(t)=v\left(t+T_{0}\right), 0 \leq t \leq \bar{T} .
\end{gathered}
$$

Proposition 3. If $\left(u_{0}, p_{0}\right)$ in $C\left(\left[0, T_{0}\right]: D(A) \times Y\right)$ denotes any solution of the inverse problem $(1)-(3)$ in $\left[0, T_{0}\right]$, then there exist $T_{1}$ in $\left[0, \min \left(T_{0}, T-T_{0}\right)\right]$ and $(u, p)$ in $C\left(\left[0, T_{0}+T_{1}\right]: D(A) \times Y\right)$ such that $(u, p)=\left(u_{0} p_{0}\right)$ in $\left[0, T_{0}\right]$, and $(u, p)$ satisfies $(1)-(3)$ in $\left[0, T_{0}+T_{1}\right]$.

Proof. It is not difficult to see that $K_{1}, K_{2}, w$ have the same properties as $F_{1}, F_{2}$, and $v$, and that $f$ and $A f$ are continuous. It follows from Theorem 1 that there exist $\left.\left.T_{1} \in\right] 0, \bar{T}\right]$ and $\left(u_{1}, p_{1}\right) \in C\left(\left[0, T_{1}\right]: D(A) \times Y\right)$, which is the unique solution of the inverse problem $(4)-(6)$ given by

$$
\begin{gathered}
u_{1}(t)=e^{A t} x+\int_{0}^{t} e^{A(t-s)} K_{1}(s, u(s), p(s)) d s+\int_{0}^{t} e^{A(t-s)} f(s) d s \\
+\int_{0}^{t} e^{A(t-s)} \int_{0}^{s} K_{2}(\sigma, u(\sigma), p(s-\sigma)) d \sigma d s, 0 \leq t \leq T_{1}, \\
p_{1}(t)=\Psi\left(t+T_{0}, w^{\prime}(t)-L A u_{1}(t)-\int_{0}^{t} L K_{2}\left(s, u_{1}(s), p_{1}(t-s)\right) d s-L f(t)\right), 0 \leq t \leq T_{1} .
\end{gathered}
$$

We have

$$
\begin{gathered}
p_{1}(0)=\Psi\left(T_{0}, w^{\prime}(0)-L A u_{1}(0)-L f(0)\right) \\
=\Psi\left(T_{0}, v^{\prime}\left(T_{0}\right)-L A u\left(T_{0}\right)-\int_{0} L F_{2}\left(s, u_{0}(s), p_{0}\left(T_{0}-s\right)\right) d s\right. \\
=p\left(T_{0}\right) .
\end{gathered}
$$


One can easily check that

$$
(u(t), p(t))=\left\{\begin{array}{l}
\left(u_{0}(t), p_{0}(t)\right), 0 \leq t \leq T_{0}, \\
\left(u_{1}(t), p_{1}(t)\right), T_{0}<t \leq T_{1}
\end{array}\right.
$$

belongs to $C\left(\left[0, T_{0}+T_{1}\right]: D(A) \times Y\right)$. It remains to show that $(u, p)$ is a solution of the inverse problem $(1)-(3)$ in $\left[0, T_{0}+T_{1}\right]$. Since $u_{1}$ satisfies (4), we can deduce that

$$
\begin{aligned}
& u^{\prime}\left(t+T_{1}\right)=u_{1}^{\prime}(t) \\
& =A u_{1}(t)+F_{1}\left(t+T_{0}, u_{1}(t), p_{1}(t)\right)+\int_{0}^{t} F_{2}\left(s, u_{0}(s), p_{1}(t-s)\right) d s \\
& +\int_{0}^{t} F_{2}\left(s+T_{0}, u_{1}(s), p_{0}(t-s)\right) d s+\int_{t}^{T_{0}} F_{2}\left(s, u_{0}(s), p_{0}\left(t+T_{0}-s\right)\right) d s \\
& =A u\left(t+T_{0}\right)+F_{1}\left(t+T_{0}, u\left(t+T_{0}\right), p\left(t+T_{0}\right)\right)+\int_{0}^{t} F_{2}\left(s, u(s), p\left(t+T_{0}-s\right)\right) d s \\
& +\int_{T_{0}}^{t+T_{0}} F_{2}\left(s, u(s), p\left(t+T_{0}-s\right)\right) d s+\int_{t}^{T_{0}} F_{2}\left(s, u(s), p\left(t+T_{0}-s\right)\right) d s \\
& =A u\left(t+T_{0}\right)+F_{1}\left(t+T_{0}, u\left(t+T_{0}\right), p\left(t+T_{0}\right)\right) \\
& t+T_{0} \\
& +\int_{0}^{0} F_{2}\left(s, u(s), p\left(t+T_{0}-s\right)\right) d s, 0 \leq t \leq T_{1} \text {. }
\end{aligned}
$$

On the other hand

$$
L u\left(t+T_{0}\right)=L u_{1}(t)=w(t)=v\left(t+T_{0}\right), 0 \leq t \leq T_{1} .
$$

Therefore we may conclude that $(u, p)$ is a solution of the inverse problem $(1)-(3)$ in $\left[0, T_{0}+T_{1}\right]$.

Proposition 4. Let $(u, p) \in C\left(\left[0, T_{\max }[: D(A) \times Y)\right.\right.$ be the maximal solution of the inverse problem (1) $-(3)$, where $0<T_{\max } \leq T$. If

$$
\max _{0<t<T_{\max }}\left(\sup _{0 \leq s \leq t}\left(\|u(s)\|_{D(A)}+\|p(s)\|_{Y}\right)\right)<+\infty
$$

then $T_{\max }=T$.

Proof. Clearly, from Proposition $2(u, p)$ can be continuously extended to a solution in 
$\left[0, T_{\max }\right]$. If $T_{\max }<T$, then, following the previous proposition, the solution in $\left[0, T_{\max }\right]$ can be extended to a solution in $\left[0, T_{\max }+\epsilon\right]$, for some $\epsilon>0$. This contradicts the maximality of $T_{\text {max }}$

Now, we will give a sufficient conditions to realize (7). For this purpose, we recall the following comparison theorem.

Theorem 2 [2]. Let $I$ be a real interval, and let $G: I \times I \times R^{+} \rightarrow R^{+}$be continuous such that $G(t, s, r)$ is monotone nondecreasing in $r$ for each $(t, s)$ in $I \times I$. Let $b$ in $C(I)$, and let $f$ in $C(I)$ denote the maximal solution of the integral equation

$$
f(t)=b(t)+\int_{t_{0}}^{t} G(t, s, f(s)) d s, t \geq t_{0}
$$

If $g \in C(I)$ is such that

$$
g(t) \leq b(t)+\int_{t_{0}}^{t} G(t, s, g(s)) d s, t \geq t_{0}
$$

then $g(t) \leq f(t), t \geq t_{0}$.

Here, by a maximal solution we mean that any other solution $h \in C(I)$ must satisfy $h(t) \leq f(t), t \geq t_{0}$.

Before stating a global existence and uniqueness result for our inverse problem, we need to modify some assumptions on $F_{1}$, and $F_{2}$.

Instead of $(H 5,2)$ and $(H 6,2)$ we suppose that there exist $G_{i}(t, r):[0, T] \times R^{+} \rightarrow R^{+}$ continuous and monotone nondecreasing in $r$ for each $t$ in $[0, T], i=1,2$, such that

$\left(H 5,2^{\prime}\right) \quad\left\|F_{1}(t, u, p)\right\|_{D(A)} \leq G_{1}\left(t,\|u\|_{D(A)}+\|p\|_{Y}\right)$,

$\left(H 6,2^{\prime}\right) \quad\left\|\int_{0}^{t} F_{2}(s, u(s), p(t-s)) d s\right\|_{D(A)} \leq \int_{0}^{t} G_{2}\left(s,\|u(s)\|_{D(A)}+\|p(s)\|_{Y}\right) d s$.

Set

$$
G(t, s, r)=M(1+k(t)\|L\|)\left(G_{1}(s, r)+(t-s) G_{2}(s, r)\right)+k(t)\|L\| G_{2}(s, r), 0 \leq s \leq t \leq T, i=0,1 .
$$

Clearly, $G(t, s, r)$ is monotone nondecreasing in $r, 0 \leq s \leq t \leq T$.

Theorem 3. Assume that $(H 1)-(H 7)$ are satisfied, where $(H 5,2)$ and $(H 6,2)$ are changed by $\left(H 5,2^{\prime}\right)$ and $\left(H 6,2^{\prime}\right)$. If the nonlinear Volterra integral equation: 


$$
r(t)=a(t)+\int_{0}^{t} G(t, s, r(s)) d s, 0 \leq t \leq T
$$

has a continuous maximal solution in $[0, T]$, then the inverse problem (1)-(3) has a unique solution in $[0, T]$.

Proof. Let $r$ denote the continuous maximal solution of the integral equation (8). Proceeding in the manner of the proof of Proposition 1, we obtain

$$
\|u(t)\|_{D(A)}+\|p(t)\|_{Y} \leq a(t)+\int_{0}^{t} G\left(t, s,\|u(s)\|_{D(A)}+\|p(s)\|_{Y}\right) d s, 0 \leq t \leq T .
$$

Thus, the condition (7) is satisfied.

The uniqueness of the global solution is just a consequence of the fact that the unique local solution allows a unique extension.

\section{STABILITY RESULT}

First of all, we give the exact assumptions under which the stability result will hold.

We assume that $(H 1)-(H 5,1),(H 6,1),(H 7)$ are satisfied, and there exist $G_{i}(t, r):[0, T] \times R^{+} \rightarrow R^{+}$continuous and monotone nondecreasing in $r$ for each $t$ in $[0, T], i=1,2$, such that

$(H 8,1) \quad\left\|F_{1}\left(t, u_{1}, p_{1}\right)-F_{1}\left(t, u_{2}, p_{2}\right)\right\|_{D(A)} \leq G_{1}\left(t,\left\|u_{1}-u_{2}\right\|_{D(A)}+\left\|p_{1}-p_{2}\right\|_{Y}\right)$, for each $\left(u_{i}, p_{i}\right)$ in $D(A) \times Y, i=1,2$, and $0 \leq t \leq T$.

$$
\begin{aligned}
& \left\|\int_{0}^{t}\left(F_{2}\left(s, u_{1}(s), p_{1}(t-s)\right)-F_{2}\left(s, u_{2}(s), p_{2}(t-s)\right)\right) d s\right\|_{D(A)} \leq \\
& \\
& \quad \int_{0}^{t} G_{2}\left(s,\left\|u_{1}(s)-u_{2}(s)\right\|_{D(A)}+\left\|p_{1}(s)-p_{2}(s)\right\|_{Y}\right) d s
\end{aligned}
$$

for each $\left(u_{i}, p_{i}\right)$ in $C([0, T]: D(A) \times Y), i=1,2$, and $0 \leq t \leq T$.

$$
\left(v(t), H_{1}(t, v(t), p)\right) \rightarrow \Phi(t, K(p)) \text { has the following property: }
$$

there exist continuous $g:[0, T] \times R^{+} \rightarrow R^{+}$, such that

$$
\left\|\Phi_{1}\left(t, w_{1}\right)-\Phi_{2}\left(t, w_{2}\right)\right\|_{Y} \leq g(t)\left(\left\|v_{1}(t)-v_{2}(t)\right\|_{Y}+\left\|w_{1}-w_{2}\right\|_{Y}\right),
$$

for each $v_{i}$ in $C([0, T]: Y), w_{i} \in Y, i=1,2$, and $0 \leq t \leq T$. 
Here, $\Phi_{i}(t, \cdot)$ denotes the inverse of the mapping $\left.K_{i}: p \rightarrow H_{1}\left(t, v_{i}(t), p\right)\right),(i=1,2)$. We set

$$
\begin{gathered}
G(t, s, r)=M(1+g(t)\|L\|)\left(G_{1}(s, r)+(t-s) G_{2}(s, r)\right)+g(t)\|L\| G_{2}(s, r) \\
0 \leq s \leq t \leq T, \quad i=0,1
\end{gathered}
$$

Theorem 4. Suppose that the assumptions listed below are satisfied for $x=x_{i}, v=v_{i}$, $i=1,2$. Let $\left(u_{i}, p_{i}\right)$ in $C([0, T]: D(A) \times Y)$ denote any solution of the inverse problem (1) $-(3)$ corresponding to $x=x_{i}, v=v_{i}, i=1,2$, and let

$$
r_{0}(t)=M(1+g(t)\|L\|)\left\|x_{1}-x_{2}\right\|_{D(A)}+g(t)\left(\left\|v_{1}(t)-v_{2}(t)\right\|_{Y}+\left(\left\|v_{1}^{\prime}(t)-v_{2}^{\prime}(t)\right\|_{Y}\right)\right.
$$

If the maximal continuous solution, given its existence, of the Volterra integral equation

$$
m(t)=r_{0}(t)+\int_{0}^{t} G(t, s, m(s)) d s, \quad 0 \leq t \leq T,
$$

satisfies the condition that there exists a constant $C>0$, not depending on $m$, such that

$$
m(t) \leq C r_{0}(t), \quad 0 \leq t \leq T
$$

then

$$
\left\|u_{1}(t)-u_{2}(t)\right\|_{D(A)}+\left\|p_{1}(t)\right\|_{Y} \leq C r_{0}(t), 0 \leq t \leq T
$$

Proof. Let $m$ denote the maximal solution of the integral equation (9), and let

$$
r(t)=\left\|u_{1}(t)-u_{2}(t)\right\|_{D(A)}+\left\|p_{1}(t)-p_{2}(t)\right\|_{Y}, 0 \leq t \leq T .
$$

It is easy to see that

$$
r(t) \leq r_{0}(t)+\int_{0}^{t} G(t, s, r(s)) d s, 0 \leq t \leq T .
$$

Using the comparison Theorem 2, we deduce that $r(t) \leq m(t)$. Hence, (11) follows from (10).

Remark. We have $G(t, s, r) \leq G(T, s, r)$. Then if $G(T, s, r)$ takes the form $G(T, s, r)=G(s) r$, the conclusion of Theorem 4.1 follows from Gronwall's inequality. 


\section{REFERENCES}

[1] Choulli, M., "Abstract inverse problem and application", J. Math. Anal. Appl.

[2] Lakshmikantham, V. and Leela, S., “Differential and Integral Inequalities”, Mathematics in Science and Engineering, Vol. 55-I, Academic Press, (1986).

[3] Lorenzi, A., “An inverse problem in the theory of materials with memory II", Universita di milano, Quaderno: 4, (1988).

[4] Lorenzi, A. and Sinestrari, E., "An inverse problem in the theory of materials with memory", J. of Nonlinear Analysis: Theory, Methods and Applications, Vol. 12, 12:1217-1333, (1988).

[5] Lorenzi, A. and Sinestrari, E., "Stability results for a partial integrodifferential equation", Proc. of the Meeting, "Volterra Integrodifferential Equations in Banach Spaces", Trento, Pitman, London, (1987).

[6] Prilepko, A.I. and Orlovskii, D.G., "Determination of evolution parameter of an equation, and inverse problems in mathematical physics I", Translations from Diff. Uravn., Vol. 21, 1:119-129, (1985).

[7] Prilepko, A.I. and Orlovskii, D.G., "Determination of evolution parameter of an equation, and inverse problems in mathematical physics II", Translations from Diff. Uravn., Vol. 21, 4:694-701, (1985). 


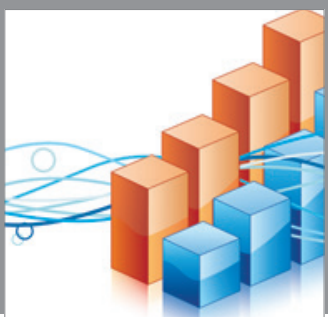

Advances in

Operations Research

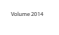

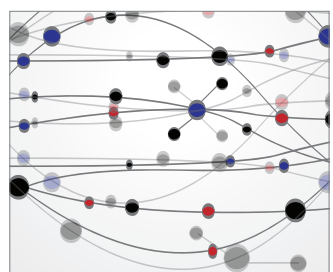

\section{The Scientific} World Journal
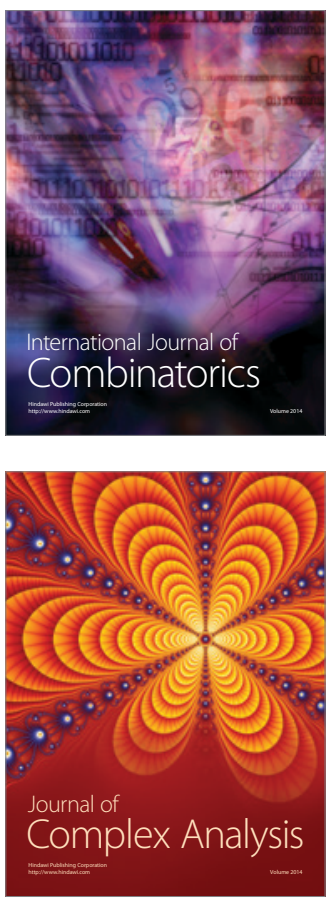

International Journal of

Mathematics and

Mathematical

Sciences
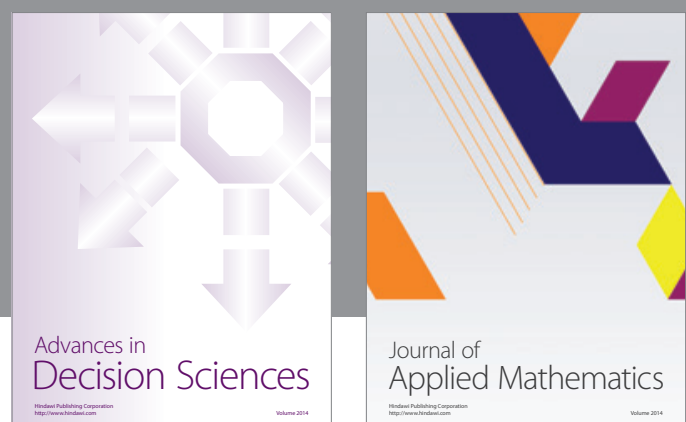

Journal of

Applied Mathematics
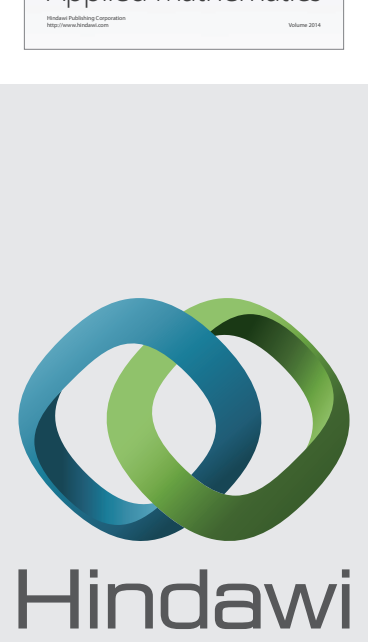

Submit your manuscripts at http://www.hindawi.com
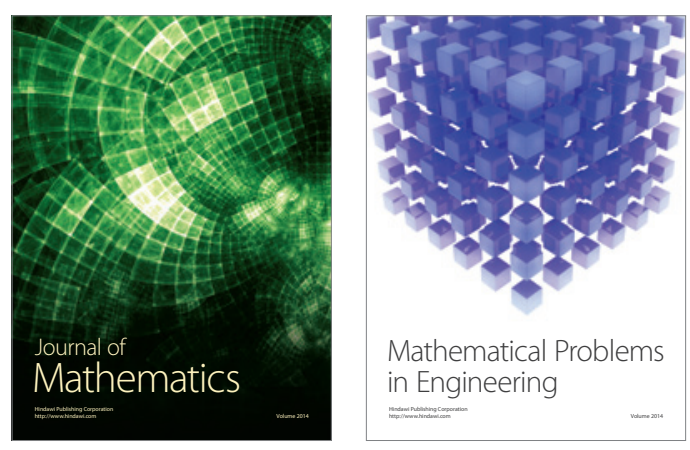

Mathematical Problems in Engineering
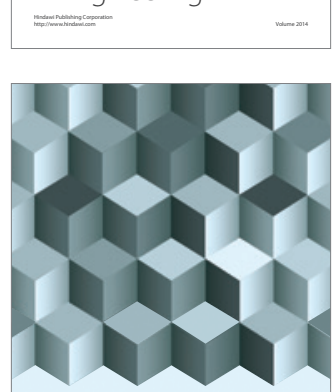

Journal of

Function Spaces
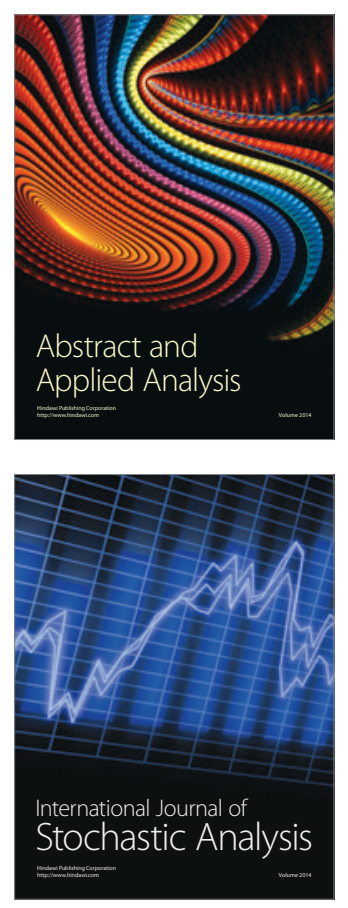

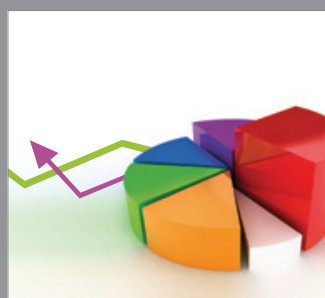

ournal of

Probability and Statistics

Promensencen
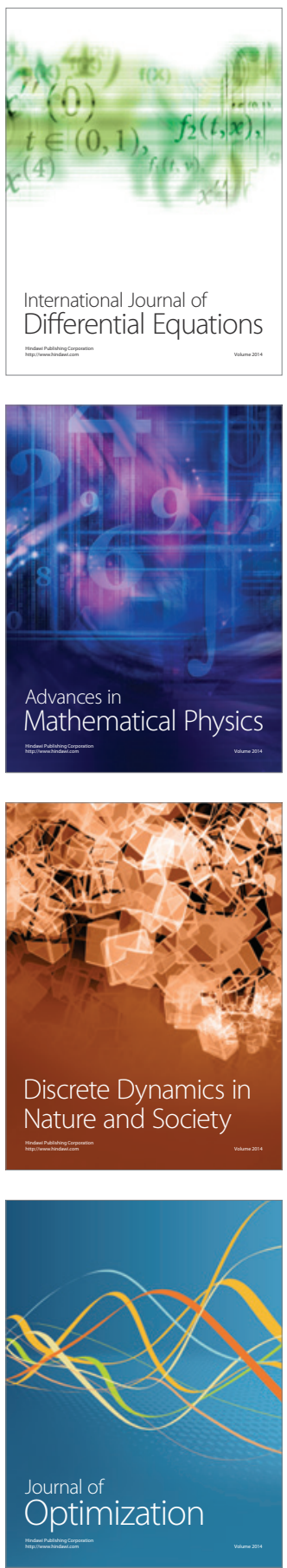